\title{
УДК 519.977
}

\section{ЗАДАЧА УПРАВЛЕНИЯ ПРОЦЕССОМ НАГРЕВА СТЕРЖНЯ С НЕИЗВЕСТНЫМИ ТЕМПЕРАТУРОЙ НА ПРАВОМ КОНЦЕ И ПЛОТНОСТЬЮ ИСТОЧНИКА ТЕПЛА ${ }^{1}$}

\author{
В. И. Ухоботов, И. В. Изместьев
}

\begin{abstract}
Рассматривается задача управления процессом нагрева стержня с помощью изменения температуры на левом конце стержня. Точные значения температуры на правом конце стержня и функция плотности тепла неизвестны, а заданы только границы областей их возможных значений. Цель процесса управления заключается в том, чтобы в фиксированный момент времени среднее значение температуры стержня находилось в заданном промежутке. Найдены необходимые и достаточные условия, которым должна удовлетворять начальная температура стержня, чтобы можно было осуществить поставленную цель при любых допустимых неизвестных функциях. Построено соответствующее управление нагревом левого конца стержня.
\end{abstract}

Ключевые слова: уравнение теплопроводности, температура, управление.

V. I. Ukhobotov, I. V. Izmest'ev. A control problem for a rod heating process with unknown temperature at the right end and unknown density of the heat source.

A control problem is considered for the process of heating a rod by varying the temperature at its left end. The exact values of the temperature at the right end of the rod and the heat density function are unknown; only the ranges of their possible values are given. The aim of the control is to ensure that the average temperature of the rod at a fixed time belongs to a given interval. We find necessary and sufficient conditions on the initial temperature of the rod under which the aim of the control can be achieved for any admissible unknown functions. The corresponding heating control at the left end of the rod is constructed.

Keywords: heat equation, temperature, control.

MSC: 49N70, 91A23

DOI: $10.21538 / 0134-4889-2019-25-1-297-305$

\section{Введение}

При изучении управляемых процессов теплопроводности, диффузии, фильтрации возникают математические задачи управления параболическими уравнениями [1-6]. На практике часто встречается задача распространения тепла в стержне, концы которого находятся при переменных управляемых температурах. Эта задача сводится к исследованию уравнения теплопроводности, граничные условия в котором зависят от управления [4-6]. В этих задачах возможны случаи, когда часть параметров уравнения и граничных условий точно не заданы.

При исследовании таких задач можно применить метод оптимизации гарантированного результата [7]. В основе этого метода лежит теория дифференциальных игр [8;9]. Помеха и неопределенность принимаются за второго игрока - противника.

В работе [10] рассмотрена задача управления процессом нагрева стержня, когда скорость изменения температуры на правом конце стержня определяется ограниченной по величине помехой. Считается, что точное значение функции плотности источника тепла неизвестно, а заданы только границы области ее значений. Управляется скорость изменения температуры на левом конце стержня. Цель процесса управления заключается в том, чтобы в фиксированный момент времени среднее значение температуры стержня находилось в заданном промежутке. Среднее значение вычисляется с помощью заданной функции.

\footnotetext{
${ }^{1}$ Работа выполнена при поддержке РФФИ (проект № 18-01-00264_a).
} 
В отличие от [10] в данной статье рассматривается случай, когда на правом конце стержня температура задается неизвестной ограниченной по величине функцией.

Задача сводится к одномерной однотипной задаче управления при наличии неопределенности. Для таких задач, рассматриваемых в рамках теории дифференциальных игр, с использованием вида альтернированного интеграла [11] построены управления игроков, которые решают поставленные перед ними задачи [12;13]. Рассмотрен пример.

\section{1. Постановка задачи}

Уравнение теплопроводности

$$
\frac{\partial T(x, t)}{\partial t}=\frac{\partial^{2} T(x, t)}{\partial x^{2}}+f(x, t), \quad 0<t<p, \quad 0<x<1,
$$

описывает распределение температуры $T(x, t)$ в однородном стержне единичной длины в зависимости от времени $t$. В начальный момент времени $t=0$ задано распределение температуры $T(x, 0)=g(x)$, где $g(x)$ - непрерывная функция. Считаем, что на левом конце стержня управляемая температура $T(0, t)$ изменяется согласно уравнению

$$
\frac{d T(0, t)}{d t}=a_{1}(t)+a_{2}(t) \xi, \quad|\xi| \leq 1 .
$$

Здесь $a_{i}(t), i=1,2$, являются функциями, непрерывными при $0 \leq t \leq p$, причем $a_{2}(t)>0$. Параметр $\xi$ является управлением.

На правом конце стержня значение температуры $T(1, t)$, зависящей непрерывно от времени $t \in[0, p]$, точно не известно. Известны только ее границы изменения

$$
b_{1}(t) \leq T(1, t) \leq b_{2}(t), \quad 0 \leq t \leq p .
$$

Здесь функции $b_{i}(t), i=1,2$, являются непрерывными при $0 \leq t \leq p$.

Относительно функции $f(x, t)$, являющейся плотностью источника тепла, известна ее оценка:

$$
f_{1}(x, t) \leq f(x, t) \leq f_{2}(x, t), \quad 0 \leq t \leq p, \quad 0 \leq x \leq 1 .
$$

Здесь функции $f_{i}(x, t), i=1,2$, являются непрерывными.

Предположение 1. Функция $f:[0,1] \times[0, p] \rightarrow \mathbb{R}$ такова, что для любых чисел $0 \leq \tau<\nu$ и непрерывных функииц $\mu_{i}:[\tau, \nu] \rightarrow \mathbb{R}, i=1,2, \beta:[0,1] \rightarrow \mathbb{R}$ таких, что выполнено условие согласования

$$
\mu_{1}(\tau)=\beta(0), \quad \mu_{2}(\tau)=\beta(1)
$$

первая краевая задача

$$
\begin{gathered}
\frac{\partial Q(x, t)}{\partial t}=\frac{\partial^{2} Q(x, t)}{\partial x^{2}}+f(x, t), \\
Q(0, t)=\mu_{1}(t), \quad Q(1, t)=\mu_{2}(t), \quad \tau \leq t \leq \nu ; \\
Q(x, \tau)=\beta(x), \quad 0 \leq x \leq 1
\end{gathered}
$$

имеет единственное решение $Q(x, t)$, непрерьвное при $0 \leq x \leq 1, \tau \leq t \leq \nu[16$, с. 237-241; 17 , c. $306-318 ; 18$, c. $200-220 ; 19$, c. $41-54]$.

Заданы числа $l \in \mathbb{R}$ и $\varepsilon \geq 0$ и непрерывная функция $\sigma:[0,1] \rightarrow \mathbb{R}$, удовлетворяющая условиям

$$
\sigma(0)=\sigma(1)=0 .
$$


Цель выбора управления $\xi(1.2)$ заключается в том, чтобы решение $T(x, t)$ уравнения $(1.1)$ удовлетворяло неравенству

$$
\left|\int_{0}^{1} T(x, p) \sigma(x) d x-l\right| \leq \varepsilon
$$

при любой непрерывной функции $T(1, t)(1.3)$ и любой непрерывной функции $f(x, t)(1.4)$, удовлетворяющей предположению 1.

\section{2. Формализация задачи}

Опишем допустимое правило формирования управления $\xi$. Оно означает, что каждому моменту времени $0 \leq \tau<p$ и каждому возможному в этот момент времени распределению температуры $T(x, \tau)$ ставится в соответствие измеримая функция $\xi:[\tau, p] \rightarrow[0,1]$. Такое правило будем обозначать как

$$
\xi(t)=N(t, T(\cdot, \tau)), \quad t \in[\tau, p]
$$

Зафиксируем разбиение

$$
\omega: 0=t_{0}<t_{1}<\ldots<t_{i}<t_{i+1}<\ldots<t_{m+1}=p
$$

отрезка $[0, p]$ с диаметром

$$
d(\omega)=\max _{0 \leq i \leq m}\left(t_{i+1}-t_{i}\right)
$$

Пусть в момент времени $t_{i}, i=\overline{0, m}$, реализовалось распределение температуры $T_{\omega}\left(x, t_{i}\right)$, $0 \leq x \leq 1$. Обозначим $\xi_{i}(t)=N\left(t, T_{\omega}\left(\cdot, t_{i}\right)\right), t \in\left[t_{i}, p\right]$. Пусть реализовались непрерывная функция $T(1, t)=\mu(t)$ (1.3) при $t_{i} \leq t \leq t_{i+1}$, у которой $\mu\left(t_{i}\right)=T_{\omega}\left(1, t_{i}\right)$, и непрерывная функция $f(x, t)(1.4)$ при $t_{i} \leq t \leq t_{i+1}, 0 \leq x \leq 1$.

Обозначим через $T_{\omega}(x, t)$ при $0 \leq x \leq 1, t_{i} \leq t \leq t_{i+1}$ решение $Q(x, t)$ задачи (1.6)-(1.8) при $\tau=t_{i}, \nu=t_{i+1}$ :

$$
\begin{gathered}
\beta(x)=T_{\omega}\left(x, t_{i}\right), \quad x \in[0,1] \\
Q(0, t)=T_{\omega}\left(0, t_{i}\right)+\int_{t_{i}}^{t}\left(a_{1}(r)+a_{2}(r) \xi_{i}(r)\right) d r, \quad t \in\left[t_{i}, t_{i+1}\right] ; \\
Q(1, t)=T(1, t), \quad t \in\left[t_{i}, t_{i+1}\right] .
\end{gathered}
$$

Отметим, что функции $\beta(x), Q(0, t)$ и $Q(1, t)$, определяемые равенствами $(2.2)$, удовлетворяют условиям согласования (1.5).

О п р е д е л е н и е 1. Управление (2.1) гарантирует выполнение поставленной цели (1.10), если для любого числа $\gamma>\varepsilon$ найдется такое число $\delta>0$, что для любого разбиения $\omega$ с диаметром $d(\omega)<\delta$ и для любой функции $f(x, t)(1.4)$, удовлетворяющей предположению 1 , при любой непрерывной функции $T(1, t)(1.3)$ выполнено неравенство

$$
\left|\int_{0}^{1} T_{\omega}(x, p) \sigma(x) d x-l\right| \leq \gamma .
$$




\section{3. Построение управления}

Обозначим через $\psi(x, \tau)$ при $0 \leq x \leq 1,0 \leq \tau \leq p$ решение следующей первой краевой задачи:

$$
\frac{\partial \psi(x, \tau)}{\partial \tau}=\frac{\partial^{2} \psi(x, \tau)}{\partial x^{2}} ; \quad \psi(x, 0)=\sigma(x) ; \quad \psi(0, \tau)=\psi(1, \tau)=0 .
$$

Из равенства (1.9) следует, что условия согласования на концах отрезка в задаче (3.1) выполнены. Поэтому решение $\psi(x, \tau)$ задачи (3.1) существует и оно непрерывно при $0 \leq x \leq 1, \tau \geq 0$ (см. $[14$, с. 44$])$.

Положим

$$
\begin{gathered}
c(t)=\frac{1}{2} \int_{0}^{1}\left(f_{2}(x, t)-f_{1}(x, t)\right)|\psi(x, p-t)| d x \geq 0, \\
c_{1}(t)=\frac{1}{2} \int_{0}^{1}\left(f_{1}(x, t)+f_{2}(x, t)\right) \psi(x, p-t) d x .
\end{gathered}
$$

Из непрерывности функций $f_{i}(x, t), i=1,2$, и $\psi(x, p-t)$ при $0 \leq x \leq 1,0 \leq t \leq p$ следует непрерывность функций (3.2) при $0 \leq t \leq p$.

Из неравенства (1.4) и из формул (3.2) имеем

$$
\int_{0}^{1} f(x, t) \psi(x, p-t) d x=c_{1}(t)+c_{2}(t) s(t), \quad|s(t)| \leq 1 .
$$

Из неравенства (1.3) следует

$$
T(1, t)=\frac{b_{1}(t)+b_{2}(t)}{2}+\frac{b_{2}(t)-b_{1}(t)}{2} \eta(t), \quad|\eta(t)| \leq 1 .
$$

Применяя лемму об выборе А.Ф. Филиппова [15], можно показать, что функции $s(t)$ и $\eta(t)$ являются измеримыми на отрезке $[0, p]$.

Введем новую переменную

$$
\begin{gathered}
z(t)=\int_{0}^{1} T(x, t) \psi(x, p-t) d x+T(0, t) \int_{t}^{p} \frac{\partial \psi(0, p-r)}{\partial x} d r \\
+\int_{t}^{p}\left(a_{1}(\tau) \int_{\tau}^{p} \frac{\partial \psi(0, p-r)}{\partial x} d r-\frac{b_{1}(\tau)+b_{2}(\tau)}{2} \frac{\partial \psi(1, p-\tau)}{\partial x}+c_{1}(\tau)\right) d \tau-l .
\end{gathered}
$$

Зафиксируем разбиение $\omega$ отрезка $[0, p]$ и управление (2.1). Подставим в формулу (3.5) реализовавшуюся функцию $T_{\omega}(x, t)$. Получим ломаную $z_{\omega}(t)$, которая удовлетворяет равенству

$$
z_{\omega}(p)=\int_{0}^{1} T_{\omega}(x, p) \sigma(x) d x-l
$$

Лемма. Для почти всех $t \in\left[t_{i}, t_{i+1}\right]$ выполнено равенство

$$
\dot{z}_{\omega}(t)=\left(a_{2}(t) \int_{t}^{p} \frac{\partial \psi(0, p-r)}{\partial x} d r\right) \xi_{i}(t)-\left(\frac{b_{2}(t)-b_{1}(t)}{2} \frac{\partial \psi(1, p-r)}{\partial x}\right) \eta(t)+c(t) s(t) .
$$


Д о к а з а т е л ь с т в о. Дифференцируя по $t$ при $t_{i}<t<t_{i+1}, i=\overline{0, m}$, равенство (3.5), получим

$$
\begin{gathered}
\dot{z}_{\omega}(t)=\theta(t)+\frac{d T_{\omega}(0, t)}{d t} \int_{t}^{p} \frac{\partial \psi(0, p-r)}{\partial x} d r-T_{\omega}(0, t) \frac{\partial \psi(0, p-t)}{\partial x}-a_{1}(t) \int_{t}^{p} \frac{\partial \psi(0, p-r)}{\partial x} d r \\
+\frac{b_{1}(t)+b_{2}(t)}{2} \frac{\partial \psi(1, p-t)}{\partial x}-c_{1}(t) .
\end{gathered}
$$

Здесь обозначено

$$
\theta(t)=\int_{0}^{1}\left(\frac{\partial T_{\omega}(x, t)}{\partial t} \psi(x, p-t)-T_{\omega}(x, t) \frac{\partial \psi(x, p-t)}{\partial \tau}\right) d x .
$$

Поскольку $T_{\omega}(x, t)=Q(x, t)$ при $t_{i} \leq t \leq t_{i+1}$ и $0 \leq x \leq 1$, то из уравнений (1.6) и $(3.1)$ имеем

$$
\theta(t)=\int_{0}^{1}\left(\frac{\partial^{2} Q(x, t)}{\partial x^{2}} \psi(x, p-t)-Q(x, t) \frac{\partial^{2} \psi(x, p-t)}{\partial x^{2}}\right) d x+\int_{0}^{1} f(x, t) \psi(x, p-t) d x .
$$

Интегрируя по частям и используя краевые условия в задачах (2.2) и (3.1), получим равенства

$$
\begin{gathered}
\left.\int_{0}^{1} \frac{\partial^{2} Q(x, t)}{\partial x^{2}} \psi(x, p-t)\right) d x=-\int_{0}^{1} \frac{\partial Q(x, t)}{\partial x} \frac{\partial \psi(x, p-t)}{\partial x} d x, \\
\int_{0}^{1} Q(x, t) \frac{\partial^{2} \psi(x, p-t)}{\partial x^{2}} d x=T(1, t) \frac{\partial \psi(1, p-t)}{\partial x}-Q(0, t) \frac{\partial \psi(0, p-t)}{\partial x}-\int_{0}^{1} \frac{\partial Q(x, t)}{\partial x} \frac{\partial \psi(x, p-t)}{\partial x} d x .
\end{gathered}
$$

Отсюда и из формулы (3.8), учитывая равенства (3.3) и (3.4), имеем

$$
\theta(t)=-\left(\frac{b_{1}(t)+b_{2}(t)}{2}+\frac{b_{2}(t)-b_{1}(t)}{2} \eta\right) \frac{\partial \psi(1, p-t)}{\partial x}+Q(0, t) \frac{\partial \psi(0, p-t)}{\partial x}+c_{1}(t)+c(t) s(t) .
$$

Подставим это выражение в формулу (3.7) и учтем равенства

$$
T_{\omega}(0, t)=Q(0, t), \quad \frac{d T_{\omega}(0, t)}{d t}=a_{1}(t)+a_{2}(t) \xi_{i}(t) .
$$

Получим требуемую формулу (3.6).

Далее, заметим, что неравенство (2.3) принимает вид

$$
\left|z_{\omega}(p)\right| \leq \gamma
$$

Обозначим

$$
\begin{gathered}
a(t)=a_{2}(t)\left|\int_{t}^{p} \frac{\partial \psi(0, p-r)}{\partial x} d r\right| \geq 0, \\
b(t)=\frac{b_{2}(t)-b_{1}(t)}{2}\left|\frac{\partial \psi(1, p-t)}{\partial x}\right|+c(t) \geq 0, \\
F(z)=\max \left(|z|+\int_{0}^{p}(b(r)-a(r)) d r ; \max _{0 \leq \tau \leq p} \int_{\tau}^{p}(b(r)-a(r)) d r\right) .
\end{gathered}
$$


Теорема 1. Пусть начальное распределение температуры $T(x, 0)=g(x)$ и число $\varepsilon \geq 0$ таковы, что выполнено неравенство

$$
F(z(0)) \leq \varepsilon .
$$

Тогда управление (2.1), определяемое формулой

$$
\xi(t)=N(t, T(\cdot, \tau))=-\operatorname{sign}\left(z(\tau) \int_{t}^{p} \frac{\partial \psi(0, p-r)}{\partial x} d r\right), \quad \tau \leq t \leq p,
$$

гарантирует выполнение поставленной чели (1.10).

Здесь и в дальнейшем sign $0=1$.

Д о к а з а т е л ь с т в о. Подставим управление (3.13) в формулу (3.6). Тогда, учитывая формулы (3.10) и (3.11), получаем

$$
\dot{z}_{\omega}(t)=-a(t) \operatorname{sign} z\left(t_{i}\right)+b(t) v(t), \quad|v(t)| \leq 1 .
$$

Здесь любое $v(t)$ с $|v(t)| \leq 1$, если $b(t)=0$ и

$$
v(t)=\frac{1}{b(t)}\left(-\frac{b_{2}(t)-b_{1}(t)}{2} \frac{\partial \psi(1, p-t)}{\partial x} \eta(t)+c(t) s(t)\right)
$$

при $b(t)>0$.

Каждая измеримая функция $v:[0, p] \rightarrow[-1,1]$ при $z_{\omega}(0)=z(0)$ определяет ломаную $z_{\omega}(t)$, удовлетворяющую уравнению (3.14). Семейство этих ломаных, определенных на отрезке $[0, p]$, является равномерно ограниченным и равностепенно непрерывным [12, с. 46]. По теореме Арцела $[20$, с. 104] из любой последовательности этих ломаных можно выделить подпоследовательность, равномерно сходящуюся на отрезке $[0, p]$. Предельная функция $z(t)$ удовлетворяет [12, теорема 8.1] неравенству

$$
|z(p)| \leq F(z(0)) .
$$

Возьмем число $\gamma>\varepsilon$. Покажем, что существует число $\delta>0$ такое, что выполнено неравенство (3.9) для любой ломаной $z_{\omega}(t)$ с диаметром разбиения $d(\omega)<\delta$.

В самом деле, допустим противное. Тогда существует последовательность ломаных $z_{\omega_{k}}(t)$ с диаметрами $d\left(\omega_{k}\right) \rightarrow 0$, у которых $\left|z_{\omega_{k}}(p)\right|>\gamma$. Можно считать, что функции $z_{\omega_{k}}(t)$ сходятся на отрезке $[0, p]$ равномерно к функции $z(t)$ (иначе перейдем к подпоследовательности). Тогда $|z(p)| \geq \gamma$. Это неравенство противоречит неравенствам (3.12) и (3.16).

Рассмотрим теперь случай, когда в (3.6) при $t_{i}<t<t_{i+1}$ реализуются функции

$$
\eta(t)=-\operatorname{sign}\left(\frac{\partial \psi(1, p-t)}{\partial x} z_{\omega}\left(t_{i}\right)\right), \quad s(t)=\operatorname{sign}\left(z_{\omega}\left(t_{i}\right)\right) .
$$

Тогда

$$
\dot{z}_{\omega}(t)=-a(t) u_{i}(t)+b(t) \operatorname{sign} z_{\omega}\left(t_{i}\right),
$$

где

$$
u_{i}(t)=-\left(\operatorname{sign} \int_{t}^{p} \frac{\partial \psi(0, p-r)}{\partial x} d r\right) \xi_{i}(t) .
$$

Выбирая произвольные измеримые функции $\left|\xi_{i}(t)\right| \leq 1$ и решая уравнение $(3.17)$ с $z_{\omega}(0)=$ $z(0)$, получим семейство ломаных $z_{\omega}(t)$.

Теорема 2. Пусть число $0 \leq \gamma<F(z(0))$. Тогда существует число $\delta>0$ такое, что $\left|z_{\omega}(p)\right|>\gamma$ для любой ломаной $z_{\omega}(t)$ с диаметром разбиения $d(\omega)<\delta$. 
Д о к а з а т е л ь с т в о. Допустим противное. Возьмем последовательность чисел $\delta_{k} \rightarrow 0$. Тогда существует последовательность ломаных $z_{\omega_{k}}(t)$, у которых диаметр $d\left(\omega_{k}\right)<\delta_{k}$ и $\left|z_{\omega_{k}}(p)\right| \leq \gamma$. Семейство ломаных $(3.17)$ с $z_{\omega}(0)=z(0)$ удовлетворяет условиям теоремы Арцела. Переходя, если нужно, к подпоследовательности, можно считать, что последовательность ломаных $z_{\omega_{k}}(t)$ сходится к $z(t)$ равномерно. Предельная функция удовлетворяет [12, теорема 8.2.] неравенству $|z(p)| \geq F(z(0))$. Стало быть, $\left|z_{\omega_{k}}(p)\right|>\gamma$ для всех достаточно больших номеров $k$. Получили противоречие.

Таким образом, наименьшее значение числа $\varepsilon$, для которого можно построить управление (2.1), гарантирующее выполнение (1.10), равно $F(z(0))$.

\section{4. Пример}

Пусть функция $\sigma(x)=\sin \pi x, 0 \leq x \leq 1$. Решением задачи (3.1) является функция

$$
\psi(x, \tau)=e^{-\pi^{2} \tau} \sin \pi x, \quad 0 \leq x \leq 1, \quad \tau \geq 0 .
$$

Из формул (3.2) находим

$$
c_{1}(t)=\frac{1}{2} e^{-\pi^{2}(p-t)} \int_{0}^{1}\left(f_{1}(x, t)+f_{2}(x, t)\right) \sin \pi x d x .
$$

Далее, из формулы (3.5) следует

$$
z(t)=e^{-\pi^{2}(p-t)} \int_{0}^{1} T(x, t) \sin \pi x d x+\frac{T(0, t)}{\pi}\left(1-e^{-\pi^{2}(p-t)}\right)+G(t),
$$

где

$$
G(t)=\int_{t}^{p}\left(\frac{a_{1}(r)}{\pi}+c_{1}(r)+\frac{\pi^{2}\left(b_{1}(r)+b_{2}(r)\right)-2 a_{1}(r)}{2 \pi} e^{-\pi^{2}(p-r)}\right) d r .
$$

Управление (3.13) представим в виде

$$
N(t, T(\cdot, \tau))=-\operatorname{sign}(z(\tau)) .
$$

\section{СПИСОК ЛИТЕРАТУРЫ}

1. Осипов Ю.С. Позиционное управление в параболических системах // Прикл. математика и механика. 1977. Т. 41, № 2. С. 195-201.

2. Короткий А.И., Осипов Ю.С. Аппроксимация в задачах позиционного управления параболическими системами // Прикл. математика и механика. 1978. Т. 42, № 4. С. 599-605.

3. Егоров А.И. Оптимальное управление тепловыми и диффузионными процессами. М.: Наука, 1978. $464 \mathrm{c}$.

4. Васильев Ф.П. Методы решения экстремальных задач. М.: Наука, 1981. 400 с.

5. Liu J., Zheng G., Ali M.M. Stability analysis of the anti-stable heat equation with uncertain disturbance on the boundary // J. Math. Anal. Appl. 2015. Vol. 428. P. 1193-1201.

6. Dai J., Ren B. UDE-based robust boundary control of heat equation with unknown input disturbance // IFAC PapersOnLine. 2017. Vol. 50, iss. 1. P. 11403-11408. doi: $10.1016 /$ j.ifacol.2017.08.1801 .

7. Красовский Н.Н. Управление динамической системой. М.: Наука, 1985. 520 с.

8. Осипов Ю.С., Охезин С.П. К теории дифференциальных игр в параболических системах // Докл. АН. 1976. Т. 226, № 6. С. 1267-1270. 
9. Охезин С.П. Дифференциальная игра сближения-уклонения для параболической системы с интегральными ограничениями на управления игроков // Прикл. математика и механика. 1977. Т. 41, № 2. C. 202-209.

10. Ukhobotov V.I., Izmest'ev I.V. The problem of controlling the process of heating the rod in the presence of disturbance and uncertainty // IFAC PapersOnLine. 2018. Vol. 51, iss. 32. P. 739-742. doi: 10.1016/j.ifacol.2018.11.458.

11. Понтрягин Л.С. Линейные дифференциальные игры преследования // Мат. сб. Новая серия. 1980. Т. 112 , № 3. С. 307-330.

12. Ухоботов В.И. Метод одномерного проектирования в линейных дифференциальных играх с интегральными ограничениями: учеб. пособие. Челябинск: Изд-во Челяб. гос. ун-та, 2005. 124 с.

13. Ухоботов В.И. Однотипные дифференциальные игры с выпуклой целью // Тр. Ин-та математики и механики Уро РАН. 2010. Т. 16, № 5. С. 196-204.

14. Годунов С.К. Уравнения математической физики. М.: Наука, 1971. 416 с.

15. Филиппов А.Ф. О некоторых вопросах теории оптимального регулирования // Вестн. МГУ. Сер. Математика, механика. 1959. Вып. 2. С. 25-32.

16. Эйдельман С.Д. Параболические системы. М.: Наука, 1964. 444 с.

17. Мизохата С. Теория уравнений с частными производными. М.: Мир, 1977. 504 с.

18. Тихонов А.Н., Самарский А.А. Уравнения математической физики. М.: Наука, 1977.735 с.

19. Ильин А.М. Уравнения математической физики: учеб. пособие. М.: Физматлит, 2009. 192 с.

20. Колмогоров А.Н., Фомин С.В. Элементы теории функций и функционального анализа. М.: Наука, 1972. 496 с.

Поступила 10.12.2018

После доработки 28.12.2018

Принята к публикации 14.01.2019

Ухоботов Виктор Иванович

д-р физ.-мат. наук, профессор

зав. кафедрой

Челябинский государственный университет

г. Челябинск

e-mail: ukh@csu.ru

Изместьев Игорь Вячеславович

канд. физ.-мат. наук, младший науч. сотрудник

Челябинский государственный университет

г. Челябинск

e-mail: j748e8@gmail.com

\section{REFERENCES}

1. Osipov Yu.S. Position control in parabolic systems. J. Appl. Math. Mech., 1977, vol. 41, no. 2, pp. 187-193. doi: 10.1016/0021-8928(77)90001-6.

2. Korotkii A.I., Osipov Yu.S. Approximation in problems of position control of parabolic systems. J. Appl. Math. Mech., 1978, vol. 42, no. 4, pp. 631-637. doi: 10.1016/0021-8928(78)90004-7.

3. Egorov A.I. Optimal'noe upravlenie teplovymi $i$ diffuzionnymi protsessami [Optimal control of thermal and diffusion processes]. Moscow: Nauka Publ., 1978, 464 p.

4. Vasil'ev F.P. Metody resheniya ekstremal'nykh zadach [Methods for solving extremal problems]. Moscow: Nauka Publ., 1981, 400 p.

5. Liu J., Zheng G., Ali M.M. Stability analysis of the anti-stable heat equation with uncertain disturbance on the boundary. J. Math. Anal. Appl., 2015, vol. 428, no. 2, pp. 1193-1201. 10.1016/j.jmaa.2015.03.073 .

6. Dai J., Ren B. UDE-based robust boundary control of heat equation with unknown input disturbance. IFAC PapersOnLine, 2017, vol. 50, no. 1, pp. 11403-11408. doi: 10.1016/j.ifacol.2017.08.1801.

7. Krasovskii N.N. Upravlenie dinamicheskoi sistemoi [Control of a dynamical system]. Moscow, Nauka Publ., 1985, 520 p.

8. Osipov Yu.S., Okhezin S.P. On the theory of differential games in parabolic systems. Sov. Math., Dokl., 1976, vol. 17, pp. 278-282. 
9. Okhezin S.P. Differential encounter-evasion game for a parabolic system under integral constraints on the player's controls J. Appl. Math. Mech., 1977, vol. 41, no. 2, pp. 194-201.

doi: 10.1016/0021-8928(77)90002-8 .

10. Ukhobotov V.I., Izmest'ev I.V. The problem of controlling the process of heating the rod in the presence of disturbance and uncertainty. IFAC PapersOnLine, 2018, vol. 51, no. 32, pp. 739-742. doi: 10.1016/j.ifacol.2018.11.458 .

11. Pontryagin L.S. Linear differential games of pursuit. Mathematics of the USSR-Sbornik, 1981, vol. 40, no. 3. pp. 285-303. doi: 10.1070/SM1981v040n03ABEH001815.

12. Ukhobotov V.I. Metod odnomernogo proektirovaniya $v$ lineinykh differentsial'nykh igrakh s integral'nymi ogranicheniyami [Method of one-dimensional projecting in linear differential games with integral constraints]. Chelyabinsk: Chelyabinsk State Univ. Publ., 2005, 124 p. ISBN: 5-7271-0725-3 .

13. Ukhobotov V.I. One type differential games with convex goal. Trudy Inst. Mat. i Mekh. UrO RAN, 2010, vol. 16, no. 5, pp. 196-204.

14. Godunov S.K. Uravneniya matematicheskoi fiziki [Equations of mathematical physics]. Moscow: Nauka Publ., 1971, 416 p.

15. Filippov A.F. On Certain questions in the theory of optimal control. J. SIAM Control Ser. A, 1962, vol. 1 , no. 1, pp. $76-84$. doi: $10.1137 / 0301006$.

16. Eidel'man S.D. Parabolic systems. Amsterdam; London: North-Holland Publ. Comp.; Groningen: Wolters-Noordhoff Publ., 1969, 469 p.

17. Mizohata S. The theory of partial differential equations. London: Cambridge Univ. Press, 1973, 490 p. ISBN: 9780521087278. Translated to Russian under the title Teoriya uravnenii s chastnymi proizvodnymi. Moscow: Mir Publ., 1977, 504 p.

18. Tikhonov A.N., Samarskii A.A. Uravneniya matematicheskoi fiziki [Equations of mathematical physics]. Moscow: Nauka Publ., 1977, 735 p.

19. Il'in A.M. Uravneniya matematicheskoi fiziki [Equations of mathematical physics]. Moscow: Fizmatlit Publ., 2009, 192 p. ISBN: 978-5-9221-1036-5/hbk.

20. Kolmogorov A.N., Fomin S.V. Elements of the theory of functions and functional analysis. (Two volumes in one, translated from the first Russian edition 1957-1961). Martino Fine Books, United States, 2012, 280 p. ISBN: 1614273049 . The 3rd edition of Russian text published in Kolmogorov A.N., Fomin S.V. Elementy teorii funktsii i funktsional'nogo analiza, Moscow: Nauka Publ., 1972, 496 p.

Received December 10, 2018

Revised December 28, 2018

Accepted January 14, 2019

Funding Agency: This work was supported by the Russian Foundation for Basic Research (projects no. 18-01-00264_a).

Viktor Ivanovich Ukhobotov, Dr. Phys.-Math. Sci., Prof., Chelyabinsk State University, Chelyabinsk, 454001 Russia, e-mail: ukh@csu.ru .

Igor' Vyacheslavovich Izmest'ev, Cand. Sci. (Phys.-Math.), Chelyabinsk State University, Chelyabinsk, 454001 Russia, e-mail: j748e8@gmail.com. 